\title{
La reflexión como eje central del desarrollo profesional
}

\author{
The reflection as a central axis for the professional development
}

Josep Roma Millán.

Departamento de Salud Pública de la Universitat de Barcelona.

En estos últimos años aparece el nuevo concepto de desarrollo profesional que viene a sustituir el clásico de formación continuada. El desarrollo profesional la incluye y pretende además valorar el aprendizaje y la mejora conseguidos mediante el progreso en la toma de decisiones complejas durante el ejercicio y la práctica de la profesión.

La reflexión se constituye como el núcleo de todas las actividades relacionadas con el desarrollo profesional y se constata la importancia de que la reflexión incorpore en su constructo los aspectos contextuales y sociales que forman parte de los problemas de salud. Se presenta el modelo de organizaciones que aprenden como ejemplo de empresas que saben aprovechar todos sus recursos y resultados para nutrir las actividades de reflexión de sus profesionales. Finalmente se comentan algunos ejemplos de actividades concretas que promueven la reflexión en el seno de dichas organizaciones, como es el caso del self-audit, el peer-review y el portafolio entre otras.

\footnotetext{
Correspondencia:

Josep Roma Millán

Consultor en Educación Médica y Salud Pública

jroma@ub.edu

C/Provença 255, $2^{\circ}$

08008 Barcelona

telf: 678651986
}

Professional development is a new concept that has arisen recently instead of the more traditional one of continuing education. Professional development goes beyond traditional activities that are included as well. Personal progress in decision taking facing complex problems is considered as crucial to understand this new concept of professional development. Reflection is the core activity in professional development. Contextual and social aspects of health problems need to integrate the construct of reflection. Learning organizations are models of companies that know how to incorporate its resources and results in order to promote reflection between their professionals. Finally some comments on main concrete activities centred on reflection are done. Self-audit, peer-review and portfolio are some of the examples exposed.

\section{INTRODUCCIÓN}

En estos últimos años han sido muchos los autores que han pretendido dar una explicación más profunda del concepto de formación continuada. Probablemente han sido los múltiples trabajos de investigación que han demostrado la escasa eficacia de la formación tradicional como instrumento de cambio los que han impulsado a responsables de for- 
mación y líderes en recursos humanos a buscar primero otras metodologías y aproximaciones, y después su conceptualización y definición, con la intención de lanzar aquellas estrategias que han permitido acercarnos a una idea de la formación mucho más próxima a la gestión de los servicios de salud ${ }^{1,2}$.

Desde la definición de la OMS en el 1975, en que se definía la formación continuada como aquella que siguen los profesionales para mantener y mejorar sus competencias, han sido muchas las miradas y aproximaciones al entorno real de la actividad profesional.

Este artículo pretende repasar estas aproximaciones centrándose en la reflexión como elemento más innovador entre los múltiples intentos de cambiar el paradigma de enseñanza hacia el de aprendizaje.

\section{HACIA UN NUEVO CONCEPTO: EL DESARROLLO PROFESIONAL}

Los primeros escarceos para intentar aproximarse a la formación continuada desde el aprendizaje se llevaron a cabo mediante el concepto de formación en servicio ${ }^{3}$. De hecho se trataba de entender el trabajo de los profesionales de la salud como un servicio al usuario o cliente, y a partir de ahí desarrollar todos aquellos aspectos que permitían aprender de la información que el propio servicio proporcionaba. Parecía lógico pues que si uno se daba cuenta de los resultados poco halagüeños del servicio prestado, se inclinara por buscar una solución en la línea de la capacitación y el aprendizaje que supusiera una mejora en el futuro de dicho servicio. De manera coherente parecía lógico que los aprendizajes serían más eficientes si se desarrollaban en el mismo servicio, valiéndose del propio acto profesional como medio para tomar consciencia del problema y también de implementar las soluciones. De hecho si había algún aspecto claro que determinaba aprendizaje y mejora en las acciones formativas, siguiendo los consejos fruto de las investigaciones antes aludidas, era precisamente el haber detectado necesidades formativas; y nada más cercano a una buena detección de necesidades que llevar a cabo la formación en el propio puesto de trabajo.

Esta noción de la formación en servicio nos acerca por otra parte a todas las estrategias de calidad 4 . Desde el desarrollo de las teorías cognoscitivas del aprendizaje asumimos que los verbos aprender y mejorar son sinónimos. No tiene demasiado sentido imaginarse situaciones den la práctica profesional en que alguien pueda aprender algo totalmente independiente de las posibles mejoras que pueda introducir en el servicio. El constructivismo, que ha realizado más tarde grandes conocimientos sobre cómo el conocimiento se crea y se forma en la persona, ha dejado intacto este aspecto, de forma que hoy podemos jugar tranquilamente con esta ecuación que iguala de hecho calidad o trabajo bien hecho, fruto de la mejora, con aprendizaje, conceptualización de aquello que debemos realizar para mejorar.

Así vemos que las similitudes entre calidad y aprendizaje son muchas. De hecho el término mejora es común a ambos conceptos, y es a partir de dicho vocablo que podemos entender las grandes coincidencias entre ambos.

Pero nuestra intención de entrada es adentrarnos más en la relación existente entre las locuciones formación continuada y desarrollo profesional continuado. El hecho de que se trata de una evolución casi natural entre uno y otro es algo asumido por todos. Tanto es así que a nuestro parecer en muchas ocasiones se acaba tratando de poco más que un cambio de etiqueta. Es decir que se siguen realizando las mismas iniciativas y desarrollos, pero bajo un paraguas que lleva un nombre distinto. En otras ocasiones $^{5}$ se teoriza un esfuerzo encomiable para reconvertir procedimientos de acreditación de aquello que anteriormente era formación continuada y que actualmente se le llama desarrollo profesional, sin hacer demasiado esfuerzo en pensar si todo lo que cabe bajo el término desarrollo es igualmente acreditable mediante un simple proceso de contar horas de dedicación.

Uno de los primeros problemas que se plantearon las entidades de acreditación en países como Estados Unidos y Canadá fue la dificultad de acreditar a partir de horas invertidas aquellas actividades que no se basaban en la formación tradicional. Es decir que todo lo que no dependía de actividades realizadas en aulas o seminarios, donde las horas lectivas eran de fácil constatación, no sabían como acreditarlo. Así ocurría con la dedicación personal o grupal al estudio, o con actividades menos formales y estandarizadas, como es el caso de las sesiones clínicas. A partir de esta dificultad parece que el concepto de desarrollo profesional lo que consigue es incorporar estas estrategias de aprendizaje a su definición, busca formas de contar las horas o el tiempo de dedicación, y con muy pocos cambios, en todo caso básicamente formales, se abre un camino que incluye la formación tradicional y que pretende ir más allá; pero si sólo se trata de esto, debemos reconocer que va muy poco más allá. 
A nuestro parecer fue Colin Coles $^{6}$ quien primero trató de distinguir entre ambos conceptos y quien realmente dio explicaciones más plausibles sobre el significado de desarrollo profesional. Creemos que realmente vale la pena la relectura de su trabajo y proponemos un cierto ejercicio de reflexión sobre su contenido.

En uno de los ejemplos Coles nos explica algo sobre los resultados de un seminario realizado sobre el tema a una serie de médicos de familia. Se trata de saber cuándo y cómo un médico toma la decisión de dar libertad a un paciente diabético para que se controle él mismo su enfermedad. Dicho de otra forma, se trata de reconocer el momento en que el paciente es autónomo. La primera pregunta que salta a la vista es: ¿puede el médico a partir del conocimiento científico existente sobre el problema en concreto tomar esta decisión? La respuesta parece clara: el conocimiento es necesario, probablemente imprescindible, pero no es suficiente. Para tomar la decisión, el médico deberá tener en cuenta otros factores, tales como el nivel cultural del paciente, su grado de conocimiento de la enfermedad, la situación familiar que le rodea, las posibilidades de acceder a ayuda profesional, el lugar donde reside...y quizá muchos otros que no somos capaces de pensar en este momento. Será en función de todas estas variables que al final tomará una decisión. Y será este proceso de toma de decisión el que realmente le hará crecer y desarrollarse.

El desarrollo profesional tiene mucho que ver con la seguridad que uno alcanza a medida que corrige su acción profesional al tomar decisiones. Obviamente que esta seguridad implica siempre algún nivel de aprendizaje, pero es evidente también que el logro va más allá del mero aprendizaje. Finalmente la sensación de seguridad se relaciona con la de mejora en el nivel de competencias y con el concepto más abstracto, pero no por ello menos utilizado, de realización personal y profesional.

La forma que tiene el profesional de alcanzar su desarrollo en el trabajo es básicamente a partir de procesos de revisión de su propia tarea. La logística de dicha revisión puede materializarse, como veremos más adelante, de formas muy diversas, pero es importante entender que este proceso de revisión es siempre el común denominador en toda actividad de desarrollo profesional.

La revisión implica la mirada hacia adentro. Es importante pues saber desarrollar la capacidad de introspección necesaria para llevarla a cabo. El profesional debe afrontar la voluntad de preguntarse a si mismo sobre aquello que probablemente no ha realizado correctamente.

Todo ello nos lleva a plantearnos la ya conocida dualidad de teorías sobre nuestro quehacer profesional que Argyris ${ }^{7}$ introdujo hace ya un tiempo. La tabla 1 resume y sintetiza los argumentos de

Tabla 1. Teorías propugnadas y teorías sobre la acción según Chris Argyris

Teoría propugnada o expuesta es aquella mediante la cual los individuos explicamos los hechos que gobiernan nuestras acciones. Generalmente es la teoría que reconocemos como válida aunque no es la que realmente utilizamos.

Teoría en uso es aquella que realmente practicamos para llevar a cabo nuestras acciones. Generalmente es la teoría que rige nuestras acciones pero que permanece escondida en nuestro subconsciente y no se hace presente.

Argyris. En resumen sabemos que los profesionales nos guiamos por dos tipos de teorías cuando tratamos de contrastar nuestra actividad: las teorías expuestas o propugnadas, que coinciden siempre con aquello que se espera de nosotros puesto que vienen a representar la forma oficial en que la sociedad espera que desarrollemos nuestra práctica; a su lado, y de forma a veces subliminal y casi desconocida, están las teorías de la acción o teorías en uso, los auténticos argumentos que motivan y mueven nuestra manera de abordar la profesión. Ocurre con frecuencia que todos pensamos que actuamos según las teorías propugnadas cuando en realidad son las teorías de la acción las que conducen nuestra actividad. Y mientras esta contradicción no pueda emerger se hace muy difícil que mejoremos $\mathrm{y}$, por tanto, aprendamos.

La revisión del trabajo realizado es la forma de romper la barrera de las teorías propugnadas y de acción. Sólo así podremos romper las llamadas barreras defensivas, aquellos argumentos y pensamientos que nos sirven para seguir creyendo que actuamos según las teorías propugnadas y que en cambio nos impiden darnos cuenta que son otros los motivos de nuestras acciones. Este proceso de revisión, que conlleva frecuentemente un replanteamiento de la forma de trabajar y por tanto unos cambios que buscan la mejora, es el que nos lleva a desarrollarnos profesionalmente. Seguro que en el proceso ha habido aprendizaje, pero el crecimiento se expresa como satisfacción de la tarea bien hecha y como realización personal a través de la mejora en la propia actividad. 


\section{LA REFLEXIÓN EN EL CORAZÓN DEL PROCESO}

La revisión como procedimiento expuesto en el apartado anterior no deja de ser una estrategia, una metodología de acción hacia la búsqueda del aprendizaje y la mejora. Aquello que está en el centro del proceso de revisión y que resulta ineludible para lograr el éxito que se propone, es la reflexión. Se trata de un proceso más complejo ya que básicamente se desarrolla en el interior de la mente humana y sólo podemos observar sus manifestaciones externas e interpretarlas para entender de qué se trata exactamente. Pero estamos de acuerdo en su esencialidad, es decir que la revisión sin reflexión no comporta precisamente aprendizaje y mejora. Puede perfectamente darse una revisión cargada de rutina que se desarrolle dentro de los cánones de las teorías propugnadas y que finalmente no aporte ningún valor añadido. Debemos pues centrar nuestro pensamiento en conocer más de cerca el qué y el cómo de la reflexión.

Para ello es necesario en primer lugar repasar lo que sabemos sobre el proceso de aprendizaje práctico o profesional. Eraut ${ }^{8}$ ya demostró ampliamente que el saber académico y el profesional son muy distintos. El conocimiento profesional sólo se puede aprender en el propio contexto, y de hecho el conocimiento académico sufre una gran transformación cuando el profesional actúa en su propio contexto y trata de adaptarlo con el objetivo de que le sea útil para solucionar los problemas que se le presentan. Nos encontramos ante una clara referencia al aprendizaje desde la experiencia como fuente de mejora evidente en las tareas profesionales. El propio Eraut, después de observar la actividad profesional tanto en maestros como en personal sanitario, explica

Tabla 2. El esquema de reflexión de la práctica según Donald Schon.

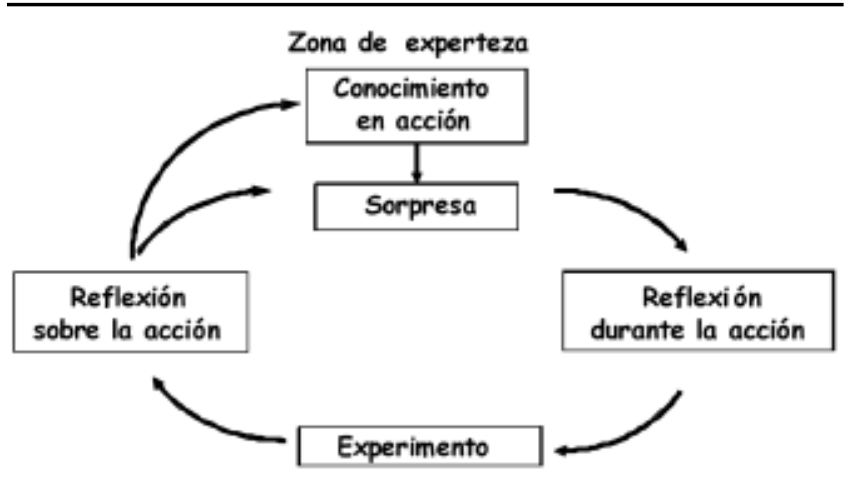

como existen cuatro estrategias consecutivas, que se extienden de lo más simple a lo más complejo, que utiliza el profesional para realizar esta traducción o adaptación del conocimiento académico al práctico: la replicación de las actividades que lleva a cabo algún otro colega más experto o competente, la aplicación de lo aprendido de nuevo a su propio entorno, la interpretación de los resultados que son fruto de las anteriores aplicaciones, y la asociación que se efectúa en situaciones conocidas similares con la finalidad de adaptarse a la propia realidad. A nuestro parecer este modelo sirve para entender cualquier proceso de especialización tal y como acaece en nuestro país. De hecho es un modelo que explica cómo hacer el tránsito desde el posicionamiento académico hacia la integración en el mundo profesional. Es pues el caso de los residentes de especialidades médicas, pero puede aplicarse a cualquier estudiante universitario que de pronto deviene un profesional y debe ejercer como tal a partir del bagaje (académico) adquirido en la universidad.

Pero para entender cómo crece un experto debemos explorar más y mejor sobre la realidad de su capacidad de reflexión. De todos es conocido el trabajo de Schon ${ }^{9}$ al respecto. La tabla 2 resume su punto de vista. Para Schon los profesionales pasan por dos momentos o instantes distintos de reflexión. Cuando ha aparecido la sorpresa, aquel aspecto de la realidad que el profesional no es capaz de resolver precisamente con su bagaje, un bagaje que, al principio, es sólo académico, se desarrolla un primer momento de reflexión rápida y superficial, muy condicionada por la presencia y persistencia del problema, que el autor denomina de reflexión durante la acción (reflection-in-action). En un segundo momento, ya después de que el profesional haya llevado a cabo alguna acción derivada de la anterior reflexión, emerge la llamada reflexión sobre la acción (reflection-on-action), un proceso de reflexión profundo y duradero que lleva al profesional a replantearse todo lo sucedido y a buscar soluciones. Dichas soluciones, que pueden materializarse en forma de diversos recursos de aprendizaje (asistir a cursos o seminarios, pero también consultar a colegas expertos, estudiar de libros y revistas científicas, o actualizarse mediante las recientes publicaciones científicas aparecidas en Medline), son las que le llevan al aprendizaje y a la mejora.

Schon nos abrió el camino, pero nos dio todavía pocas pistas de cómo se producía este auténtico momento de crecimiento que llamaba reflection-onaction. Fook ${ }^{10}$ ha trabajado con más detalle el signifi- 
cado de la reflexión y creemos que ha aportado luz al tema. La idea básica de Fook es que el profesional no trabaja en solitario, que sólo podemos entender su labor a partir de un constructo que mezcla la actuación individual como profesional con la comunidad o sociedad a la que sirve. Dicho de otra forma, los problemas (sorpresas en el lenguaje de Schon) que afronta un médico o una enfermera no pueden ser entendidos exclusivamente como problemas técnicos. Si así fuere, bastaría con el conocimiento académico para resolverlos. Son problemas que se manifiestan en un contexto determinado, junto a una serie de exigencias sociales, y cuya expresión tiene siempre un componente social evidente. El ejemplo descrito anteriormente de la toma de decisión sobre la autonomía de cada paciente sigue siendo bueno para entender este constructo, y puede evidentemente ser utilizado para cualquier caso de determinar autonomía. La tabla 3 describe la secuencia de pasos que según Fook sigue un profesional para aprender de la experiencia cuando tiene en cuenta precisamente la necesidad de validación social del proceso. Se trata de una aportación fundamental puesto que distingue bien lo que sería un proceso de adaptación y transformación del aprendizaje académico (siguiendo a Eraut) cuando alguien empieza su vida profesional, de lo que sería un proceso de desarrollo y aprendizaje cuando este alguien ya se planeta como meta llegar a ser un experto y por tanto, de forma ineludible, debe tener en cuenta sobre todo el contexto y la realidad social donde ha de solucionar sus problemas.

Tabla 3. Proceso de reflexión profesional según Jan Fook

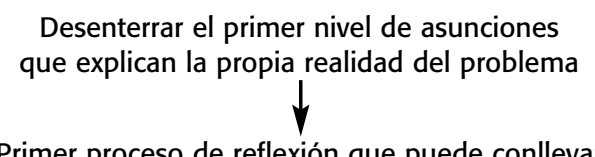

Primer proceso de reflexión que puede conllevar la emergencia de otras asunciones

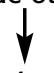

Establecimiento de conexiones entre las asunciones y hechos concretos de la vida profesional

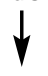

Las asunciones son evaluadas (validadas) o contrastadas frente a la experiencia actual y los valores que la conforman siguiendo la lógica del propio contexto

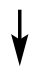

Las viejas asunciones son reformuladas según el modelo de práctica profesional que se asume desde la propia acción

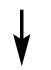

Se introducen cambios en la práctica profesional de acuerdo con lo aprendido hasta el momento

\section{HACIA UN MODELO DE EMPRESA: LAS ORGANIZACIONES QUE APRENDEN}

El reto que nos queda por desgranar es el que se plantea desde las organizaciones o instituciones sanitarias cuando se intenta modelar un sistema de formación que encaje precisamente con todo lo expuesto anteriormente. Es decir que sea un modelo basado en el aprendizaje y la mejora, que esté bien conectado con las estrategias de mejora de calidad, que de alguna forma promueva la reflexión de sus profesionales y trabajadores. La solución, aunque reconocemos que más teórica que práctica, está en el modelo de las learning organizations, o organizaciones que tienden a orientarse hacia el aprendizaje.

La idea de este modelo surgió con Senge ${ }^{11}$ a principios de la década de los noventa del pasado siglo, con el propósito de tratar de explicar cómo algunas grandes empresas norteamericanas sobrevivieron a la llamada crisis del petróleo y al surgimiento de la economía del conocimiento.

La propuesta de Senge se basa en terminar con los modelos centrados en los grandes planes de formación dirigidos por los no menos importantes departamentos de formación o de recursos humanos. En primer lugar se centra en el aprendizaje y la mejora destacando que este es el objetivo de la empresa para con sus profesionales. En segundo lugar se pregunta donde se puede ejercitar el aprendizaje y se da cuenta que cualquier estrategia debe basarse en la experiencia, en el propio trabajo y sus resultados. Es aquí donde, quizá sin saberlo, Senge entronca con los posicionamientos de los autores que hemos citado y que trataron de aportar valor añadido al concepto de formación llevándolo al terreno del desarrollo profesional y de la reflexión de la práctica.

En el esquema que nos propone presenta la organización que aprende como un compendio de cinco aspectos a tener en cuenta: 1) el pensamiento sistémico, que consiste en saber entender el funcionamiento de la organización como una red de servicios, departamentos, personas y funciones que se entrelazan y se relacionan comúnmente; 2) la visión compartida, que pretende ser una estrategia de conceptualización de la organización que es común a todos los trabajadores; 3 ) el trabajo en equipo, algo que ya hoy nadie duda que es absolutamente necesario para la buena marcha de la empresa, puesto que la suma de profesionales resulta sinérgica si se logra este objetivo; 4) el desarrollo integral de la persona, puesto que a diferencia de lo que ocurría en la déca- 
da anterior, sabemos que debe existir un equilibrio entre vida personal, familiar y profesional para asegurar el éxito y el crecimiento de todos; 5) el cambio en los modelos mentales, que es de hecho lo que Senge llama la quinta disciplina, la que es propia y nueva en el concepto de organización que aprende, y que plantea precisamente la eliminación de las barreras y prejuicios que los miembros de la organización ponen por delante y les impide el cambio. Como vemos esta idea de cambiar modelos mentales enlaza bien con los posicionamientos de Argyris antes mencionados, y deberá servirse de la actividad reflexiva para poder ponerse en práctica.

En suma podemos decir que una organización que aprende es aquella que se plantea que todo lo que ocurre en su seno, todos los resultados fruto de su funcionamiento, deben ser aprovechados para el aprendizaje y mejora de los profesionales. Se entiende, claro está, que el aprovechamiento que realizarán los profesionales tiene su vertiente individual, pero aún más importante, tiene su otra vertiente de equipo. Vemos así que volvemos al discurso anterior: se trata de poner los resultados, es decir la experiencia, a disposición de los profesionales, para conseguir que ellos realicen su proceso de reflexión que les permita desarrollarse, aprender y mejorar. Y siendo coherentes con Fook, también comprendemos que la reflexión debe partir asimismo del constructo social en que está inmerso el profesional, constructo que se desprende de la visión de la empresa, su misión y objetivos, y las necesarias relaciones con el entorno que dan coherencia a su existencia.

Es Argyris quien nos da la mejor pista sobre estrategias a seguir para relacionar la actividad reflexiva y el desarrollo de una organización que aprende. Según él, podemos observar dos ciclos o bucles de aprendizaje en este tipo de organizaciones. El primero se forma cuando los profesionales se plantean soluciones a problemas técnicos expresados generalmente por los clientes, y lo hacen desde la perspectiva de su falta de capacitación. Se trata de un bucle sencillo que probablemente se resuelva con estrategias simples de reflexión que no ponen en duda el modelo. El segundo se crea cuando los profesionales se plantean las soluciones de los mismos problemas desde la perspectiva de la misión, objetivos y valores de la organización. Ahí surge el reto de la validación social del proceso de reflexión y es cuando el bucle de aprendizaje es más complejo y precisa del trabajo en equipo y la confrontación con el modelo de funcionamiento. Se trata finalmente de un proceso más acorde con el modelo de Fook.

\section{ALGUNOS EJEMPLOS DE IMPLEMENTACIÓN}

Finalmente hemos considerado que no podíamos acabar este artículo sin tratar de aterrizar y hablar de aspectos concretos que todo el mundo pueda imaginarse aplicándolos. Es por ello que hemos elaborado un listado de actividades que encajan plenamente con el paradigma expuesto. Es decir que son propuestas que responden a aquello que una organización que aprende puede poner en marcha para conseguir el desarrollo de sus profesionales a partir de procesos de reflexión desde la experiencia o práctica real. Vaya por delante que el listado no pretende ser exhaustivo ni mucho menos; creemos sinceramente que es la imaginación de los responsables de formación y de desarrollo aquello que se debe estimular. En este terreno no hay estándares fijos sino experiencias que serán distintas en cada caso. Incluso cada uno de los supuestos de la relación que comentamos brevemente puede ser implementado de manera diversa. Antes de pasar a la relación un breve comentario sobre el feedback; no lo detallamos como un método o instrumento aislado puesto que entendemos que se trata de la base de cualquiera de los que comentamos seguidamente. Es decir que en cualquiera de ellos el feedback está siempre presente porque es precisamente el núcleo del proceso.

Self-audit. Es un término inglés que significa autoevaluación. Se trata de un proceso que comienza con la fijación de indicadores en forma de estándares y criterios de calidad, le sigue una revisión individual en los propios registros clínicos de dichos indicadores, una reflexión personal sobre aquellos aspectos correctos y mejorables, y finalmente una sesión con alguien que da feedback sobre el proceso.

Peer-review. Lo podemos traducir como revisión entre iguales. Se utiliza la misma metodología que en el self-audit, pero en este caso la evaluación de registros se realiza de forma cruzada entre dos colegas. Una vez realizada la comprobación de indicadores en los registros del otro, se lleva a cabo un feedback mutuo entre el par de iguales implicado.

Observación directa. Se trata de buscar un mecanismo por el cual alguien observa la práctica profesional de otro bajo la guía de un listado de comprobación donde se puede anotar aquello que se observa realizar. La observación in situ a veces es difícil de llevar a cabo por problemas logísticos, pero se puede sustituir por vídeo grabaciones que pueden ser observadas con posterioridad. El feedback puede ser dado finalmente por un colega o igual, o por un mentor o experto externo. Ambos modelos 
son factibles y responden a objetivos distintos en la organización.

Observación de situaciones simuladas. Aunque generalmente en el ámbito profesional se prefiere partir de la propia realidad, a veces puede ser conveniente utilizar simulaciones para invitar a los profesionales a actuar y ser observados. La ventaja de la simulación es que se trata de creaciones ad hoc que pueden ser útiles para valorar situaciones poco frecuentes o particularmente complejas. Se puede trabajar tanto con enfermos simulados como con casos clínicos desarrollados a partir de programas informáticos. El primer ejemplo nos sirve para explorar aspectos como la comunicación y la capacidad de obtener información, mientras que en el segundo exploramos la capacidad de tomar decisiones y el proceso de razonamiento implícito.

Discusión de resultados asistenciales. Se trata de un proceso emparentado con las técnicas de análisis y mejora de calidad. Los resultados se presentan generalmente a nivel de grupo (servicio, departamento, centro, equipo de atención, etc.) y es el grupo entero quien se plantea como mejorarlos y qué estrategias de aprendizaje llevar a cabo para conseguirlo. Aquí se trata de explotar al máximo las potencialidades de aprendizaje en grupo siguiendo estrategias propias del ABP (Aprendizaje Basado en Problemas).

Portafolio. Lo citamos en última instancia porque se trata de un "instrumento de instrumentos". En realidad su objetivo es coleccionar evidencias de la mejora o aprendizaje de uno mismo como profesional. Pretende además estimular la reflexión a partir del análisis detallado de dichas evidencias y mediante una estructura lógica que lo facilite. El portafolio puede por tanto utilizar y combinar todos los instrumentos o actividades citados arriba e incluso muchos otros que pueden resultar útiles para su propósito.

\section{BIBLIOGRAFÍA}

1. Davis DA, Thomson MA, Freemantle N, Wolf FM, Mazmanian $\mathrm{P}$, Taylor-Vaisey A. Impact of formal continuing medical education. Do conferences, workshops, rounds and other traditional continuing education activities change physician behaviour or health care outcomes? JAMA 1999; 282: 867-874.

2. Oxman Ad, Thomson MA, Davis DA, Haynes RB. No magic bullets: a systematic revuew of 102 trials of interventions to improve professional practice. CMAJ 1995; 153: 1423-1431.

3. Le Boterf G. Ingénierie et evaluation des competentes. Editions d'organisation 2002.

4. Fleisher DS, Brown CR, Zeleznick C, Escovitz GH, Omdal C. The mandat project: institutionalizing a system of patient care quality assurance. Pediatrics 1976; 57:775-782.

5. Bruguera M, Gual A. Retos del desarrollo profesional continuado y la regulación de la profesión médica. Educación Médica 2006; 9: 193-200.

6. Coles C. Aproaching professional development. Journal of Continuing Education of Health Professions 1996; 16: 152158.

7. Argyris C. Knowledge for action. San Francisco. Jossey-Bass 1993.

8. Eraut M. Developing professional knowledge and competence. The Falmer Press, 1994.

9. Schon DA. Educating the reflective practitioner. San Francisco: Jossey Bass, 1987.

10.Fook J. Beyond reflective practice: reworking the "critical" in critical reflection. Standing conference on University teaching and research in the education of adults. Leeds 2006.

11.Senge P. The fifth discipline. Doubleday. New York, 1990. 